\title{
A Non-Linear Mixed Spectral Finite-Difference 3-D model for planetary boundary-layer flow over complex terrain
}

\author{
W. Weng and P. A. Taylor \\ Department of Earth and Space Science and Engineering, York University, 4700 Keele Street, Toronto, \\ Ontario, M3J 1P3, Canada
}

Received: 31 December 2010 - Revised: 15 March 2011 - Accepted: 27 March 2011 - Published: 1 April 2011

\begin{abstract}
The Non-Linear Mixed Spectral Finite-Difference (NLMSFD) model for surface boundary-layer flow over complex terrain has been extended to planetary boundary-layer flow over topography. Comparisons are made between this new version and the surface layer model. The model is also applied to simulate an Askervein experimental case. The results are discussed and compared with the observed field data.
\end{abstract}

\section{Introduction}

The Mixed Spectral Finite-Difference (MSFD) model was originally developed by Beljaars et al. (1987). It is based on the idea that the topography produces a perturbation to a steady, neutrally stratified, non-evolving flow over horizontally homogeneous flat terrain. A number of efforts have been made to improve the model calculation of the turbulent boundary-layer flow over complex terrain. Ayotte et al. (1994) evaluated the model predictions with a number of different closure schemes which range from the simple firstorder $\kappa-Z$ closure to the full second-order closure. Xu and Taylor (1992) and Xu et al. (1994) made the non-linear extension of the model by including all the neglected terms. In the non-linear version of the MSFD model (NLMSFD), the model equations were solved iteratively. Another model improvement is the extension to the stable boundary layer (MSFD-STAB, see Weng et al., 1997).

Although the MSFD and NLMSFD models have been improved since the late 80 's, these models can only formally apply to the surface-layer flow due to the model assumption that upwind or zero-order profiles of mean and turbulent variables are simple logarithmic surface-layer profiles, e.g., wind speed is logarithmic, shear stresses are constant and the effect of Coriolis force is absent. Ayotte and Taylor (1995) made the first effort to extend the model to the planetary boundary-layer flow with the full second-order turbulence closure (MSFD-PBL) but it was still a linear model.

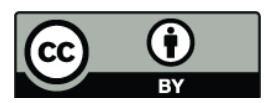

Correspondence to: W. Weng

(wweng@yorku.ca)

\section{The model}

\subsection{The model equations}

For the PBL boundary-layer flow, the model uses the Reynolds-averaged equations for steady-state, neutrally stratified incompressible flow. They are, in tensor notation including use of the summation convention,

$$
\begin{aligned}
U_{j} \frac{\partial U_{i}}{\partial x_{j}} & =-\frac{1}{\rho} \frac{\partial p}{\partial x_{i}}+f \epsilon_{i j 3}\left(U_{j}-U_{\mathrm{g} j}\right)-\frac{\partial\left\langle u_{i} u_{j}\right\rangle}{\partial x_{j}} \\
\frac{\partial U_{i}}{\partial x_{i}} & =0
\end{aligned}
$$

where $U_{i}$ and $u_{i}$ are the $i$-th component of the mean and turbulent flow respectively; $f$ is the Coriolis parameter; $\epsilon_{i j 3}$ is the alternating unit tensor; and angle bracket $(\langle\rangle)$ denotes an ensemble mean. The pressure gradient force consists of a nonhydrostatic mesoscale pressure component, $P$, and a synoptic-scale component, $f \epsilon_{i j 3} U_{\mathrm{g} j}$, where $U_{\mathrm{g} j}$ is the $j$-th component of the geostrophic wind.

To close the system of the equations, a turbulent closure scheme is needed. Weng and Taylor (2003) have shown that the so-called simple $E-\ell$ turbulence closure scheme performs quite well in modelling the PBL flow in most atmospheric conditions compared with more sophisticated schemes. The $E-\ell$ turbulence closure is a $1 \frac{1}{2}$-order scheme in which the prognostic equations for the turbulent kinetic energy $(E)$ and a diagnostic equation for the turbulent length scale $(\ell)$ are used. The turbulent fluxes are locally related to mean vertical gradients and an eddy diffusivity, see Weng and Taylor (2003) for details. 


\subsection{Numerical scheme and boundary conditions}

As with the previous versions of MSFD/NLMSFD model, the model equations are transformed from the original standard right-hand coordinate system $(x, y, z)$ with $z$ in the vertical direction to a new system $(X, Y, Z)$, by using a terrainfollowing coordinate transform. In addition, to ensure sufficient resolution near the surface and to resolve strong gradients, a log-linear coordinate transform is further used for the vertical coordinate $Z$.

Fourier transformation is performed in the horizontal directions to the coordinate transformed governing equations. The model variables are decomposed into an unperturbed or zero-order part, independent of $x$ and $y$, corresponding to equilibrium flow over uniform flat terrain and a perturbation part due to topographic forcing. Collecting the first-order perturbation terms and solving the resulting system of equations, forms the linear version of the model (MSFD-PBL). Treating the neglected high-order terms as the source terms and solving the resulted equations iteratively leads to the NLMSFD-PBL.

After all these transformations, equations are discretized. A staggered vertical grid is used, where $U$-grid points are located midway between neighboring $W$-grid points. Variables stored at $U$-grid points are $U, V$, and $P$; while $W$ and turbulent quantities are at $W$-grid points. The lower and upper boundaries are at $W$-grid locations. The resulting set of difference equations are solved using a block $\mathbf{L} \mathbf{U}$ factorization algorithm (Karpik, 1988).

The surface boundary conditions used are a non-slip condition for velocity, the vertical derivative of the perturbation pressure is zero and local equilibrium condition for the turbulent quantities. At the upper boundary, the effects of the topography vanish. We set the perturbations of mean variables and the vertical derivatives of turbulent quantities to zero. The model uses periodic boundary conditions in $X$ and $Y$ directions.

\subsection{Upstream profiles}

The upstream or undisturbed profiles for the current PBL model are the results of an integration of a 1-D unsteadystate form of the model equations to quasi-steady state. For the given conditions of the site location $(f)$, the geostrophic wind speed $\left(U_{\mathrm{g}}, V_{\mathrm{g}}\right)$ and the surface roughness length $\left(z_{0}\right)$, we can obtain the necessary equilibrium profiles by running the 1-D PBL model of Weng and Taylor (2003). This model will be used for providing all required upstream profiles in our simulations.

\section{Results and discussions}

Model runs have been carried out for boundary-layer flow over an idealized isolated 3-D terrain and the Askervein Hill

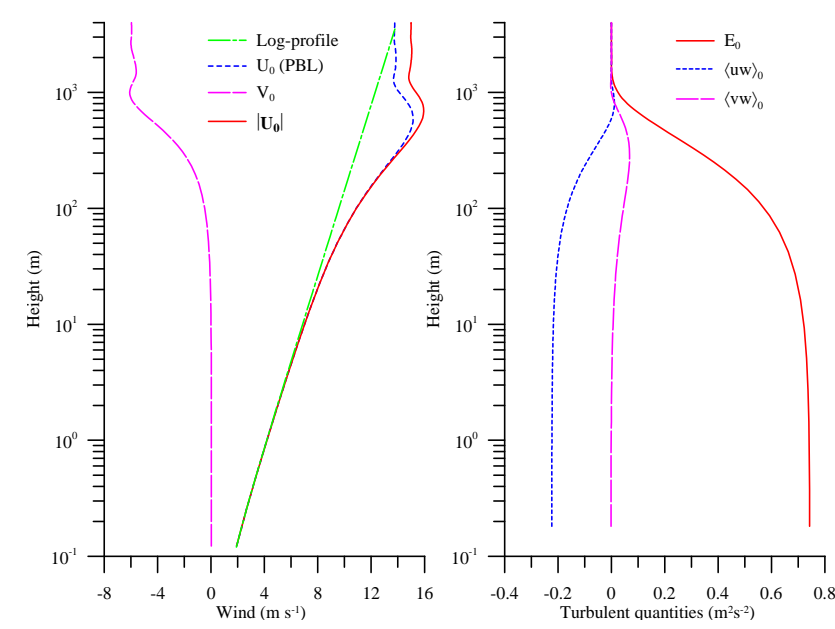

Figure 1. Initial input profiles of $U_{0}, V_{0},\left|\mathbf{U}_{0}\right|, E_{0},\langle u w\rangle_{0}$ and $\langle v w\rangle_{0}$, which is the result of 1-D PBL model runs of Weng and Taylor (2003) for the given condition of $z_{0}=0.03 \mathrm{~m}, f=10^{-4} \mathrm{~s}^{-1}$, $\left(U_{\mathrm{g}}, V_{\mathrm{g}}\right)=(13.77,-5.95) \mathrm{m} \mathrm{s}^{-1}$, neutral thermal stratification. Logarithmic wind profile for the surface layer model is also inclcuded.

- the site of a detailed and much referenced field study of boundary-layer flow over low hills in the 1980s.

\subsection{Flow over idealized terrain}

For the idealized case, an "isolated", "cosine-squared" terrain surface is used, which is described by

$z_{s}(x, y)= \begin{cases}h \cos ^{2}(\pi r / \lambda), & \text { for } \quad r<\lambda, \\ 0, & \text { for } \quad r \geq \lambda,\end{cases}$

where $r=\sqrt{x^{2}+y^{2}}$ and the maximum slope is $\pi h / \lambda$. For our test case, the values of $h=75 \mathrm{~m}$ and $\lambda=1500 \mathrm{~m}$ are used and the maximum slope is 0.157 . Our computational domain is set as $(X, Y) \in[-3000,3000] \mathrm{m}$ and $4000 \mathrm{~m}$ in the vertical and $129 \times 129 \times 101$ grid points are employed.

Figure 1 shows the initial background profiles of $U_{0}, V_{0}$, $\left|\mathbf{U}_{0}\right|, E_{0},\langle u w\rangle_{0}$ and $\langle v w\rangle_{0}$. This is the result of the 1-D PBL model runs of Weng and Taylor (2003) for surface roughness $z_{0}=0.03 \mathrm{~m}$, Coriolis parameter $f=10^{-4} \mathrm{~s}^{-1}$, geostrophic wind is constant with height and set to $\left|\mathbf{U}_{\mathrm{g}}\right|=15 \mathrm{~m} \mathrm{~s}^{-1}$ (the components are selected as $\left(U_{\mathrm{g}}, V_{\mathrm{g}}\right)=(13.77,-5.95) \mathrm{m} \mathrm{s}^{-1}$ so that the near surface (at $z=10 \mathrm{~m}$ ) wind direction is approximately 0 ) and we assume neutral thermal stratification. This leads to the surface friction velocity, $u_{*} \approx 0.47 \mathrm{~m} \mathrm{~s}^{-1}$, which is used to calculate the logarithmic wind profile for the surfacelayer model runs. As can be seen clearly from the figure, the PBL has a near logarithmic mean wind profile associated with a well developed constant stress layer to a depth of about $40 \mathrm{~m}$ (where TKE is about $90 \%$ of its surface value). Above this surface layer, the mean wind profiles show a smooth blending to geostrophic values at the upper boundary of the model. The PBL has a depth of about $900 \mathrm{~m}$. The turbulent 


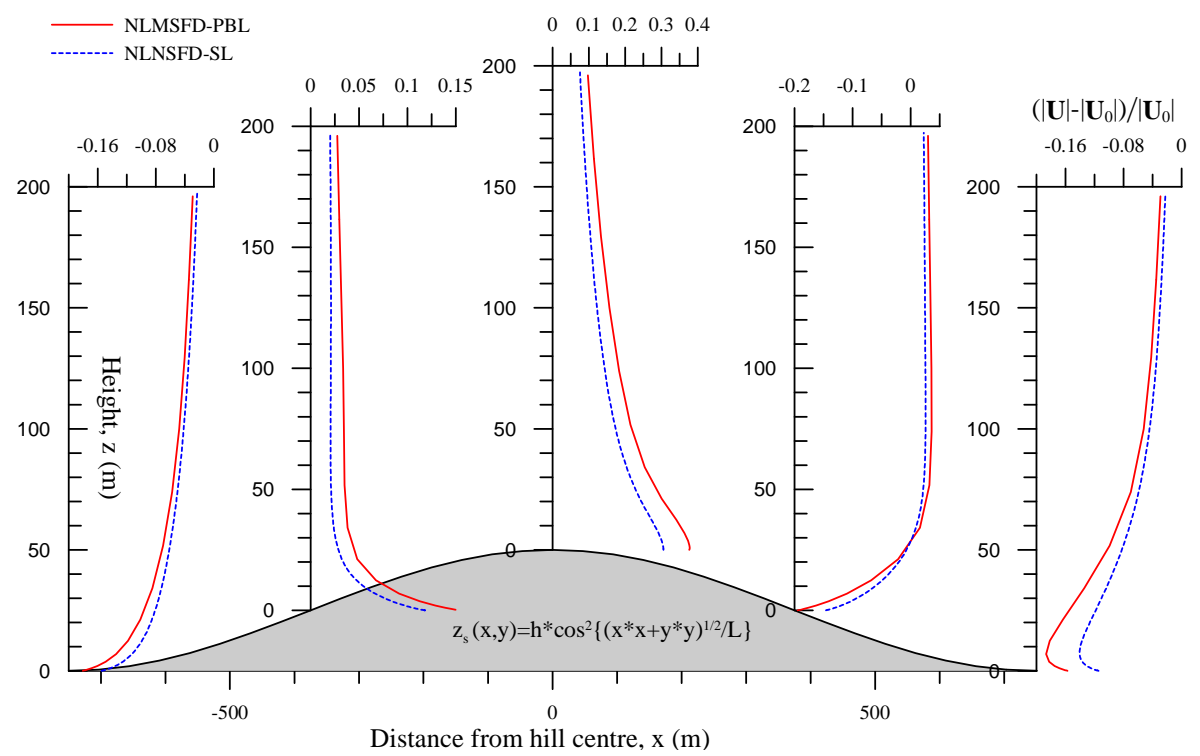

Figure 2. Comparison of vertical fractional speed-up profiles at five different locations along the central line $(y=0)$ of a circular cosinesquared hill from NLMSFD-PBL and NLMSFD-SL model runs.

quantities (TKE and shear stress) decrease appreciably over the depth of the boundary layer and diminish to near zero in the absence of shear at the top of the PBL. There are also supergostrophic winds around $z=650 \mathrm{~m}$ and the wind vector has Ekman spiral behaviour.

Figure 2 shows the comparison of vertical fractional speed-up $(\Delta S)$ profiles at five different locations along the central line $(y=0)$ from NLMSFD-PBL and NLMSFD-SL model runs. $\Delta S$ is defined as the difference between the local $(|\mathbf{U}(X, Y, Z)|)$ and the upstream $\left(\left|\mathbf{U}_{0}(Z)\right|\right)$ wind speeds divided by the upstream wind speed. It can be clearly seen that the PBL version of the model predicts larger speed-up around the hill top areas and larger wind reduction at both upwind and downwind hill foot areas than the surface-layer model. Even at this lower maximum slope of 0.157 , the difference between the two models is apparent.

\subsection{Flow over Askervein Hill}

Askervein is a $116 \mathrm{~m}$ high (126 m above the sea level) hill on the west coast of South Uist, one of the islands of the Outer-Hebrides (Scotland). For our model computation, the terrain data was originally prepared by Walmsley, see Walmsley and Taylor (1996). There were a lot of very small terrain features which lead to a very noisy FFT (Fast Fourier Transform) field in the original, so-called Map B. For our model runs, a 9-point smoothing was used. There is a slightly decrease in amplitude in the resulting topography. The maximum height of the hill becomes $115.73 \mathrm{~m}$ and the maximum slope is about 0.39 , see Fig. 3, where three tower lines and $10 \mathrm{~m}$ measurement locations are overlaid. Line-A and LineAA go through the hill top (HT) and hill centre $(\mathrm{CP})$ respec-

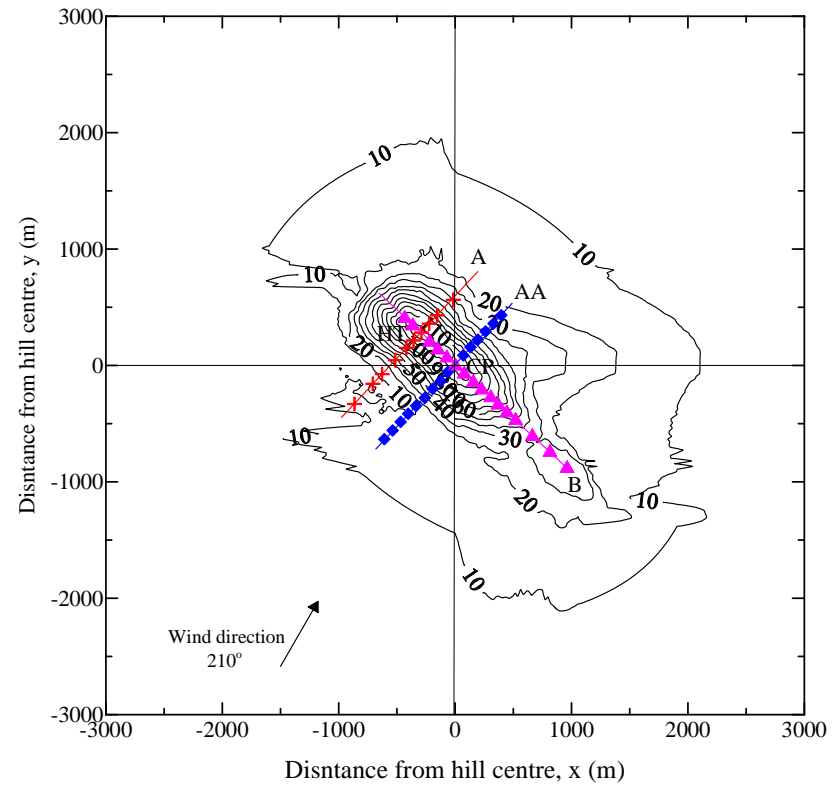

Figure 3. Computational domain of the Askervein hill and measurements tower along the A-line, AA-line and B-line.

tively and are perpendicular to the ridge, and Line-B goes through $\mathrm{HT}$ and $\mathrm{CP}$ along the hill ridge.

Our computational grid consists of $129 \times 129$ points uniformly distributed over an area of $6000 \times 6000 \mathrm{~m}^{2}$. This makes the resolution $46.875 \mathrm{~m}$ and the domain sufficiently large to avoid upstream effects due to the periodic boundary conditions. The model run is for the wind direction of $210^{\circ}$, $-13^{\circ}$ from the $223^{\circ}$ orientation of lines A and AA. The surface roughness is assumed uniform and taken as $z_{0}=0.03 \mathrm{~m}$. 


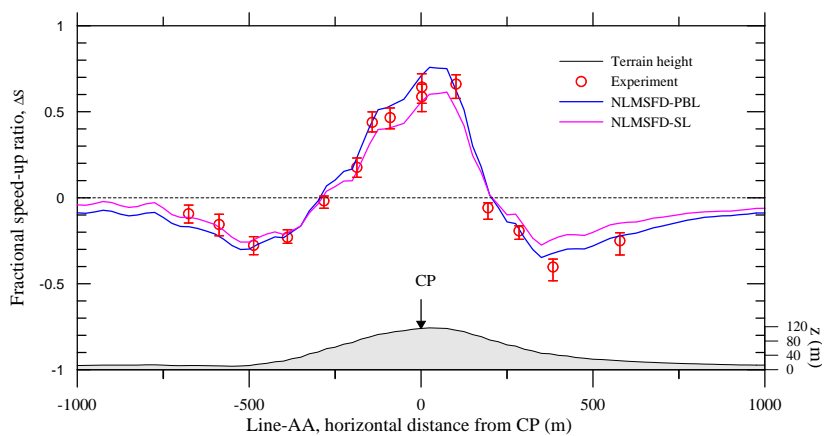

Figure 4. Fractional speed-up ratio, $\Delta S$, for flow over Askervein hill at a height of $10 \mathrm{~m}$ above topography along line AA. Comparison of model results and experimental data.

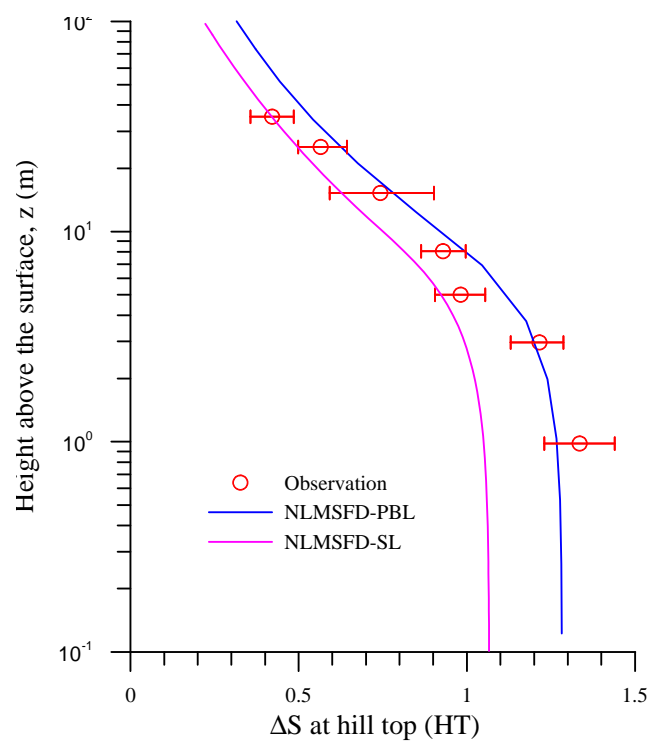

Figure 5. Vertical profile of fractional speed-up ratio, $\Delta S$, at the hill top (point HT) of Askervein hill. Comparison of model results and experimental data.

To closely match the observational data, $\left|\mathbf{U}_{\mathrm{g}}\right|=$ $19.25 \mathrm{~m} \mathrm{~s}^{-1}$ is used. Again the 1-D PBL model of (Weng and Taylor, 2003) is used to compute the upstream profiles, which are very similar to those in Fig. 1.

Model results of fractional speed-up ratio at $10 \mathrm{~m}$ along the line AA together with observational data are shown in Fig. 4. The NLMSFD-PBL predicts slightly smaller values of $\Delta S$ over the upwind hill foot and lee side ares and slightly larger value over hill top (HT) area and agrees better with the measured data than NLMSFD-SL results. Figure 5 shows the comparison of vertical profiles of $\Delta S$ at HT. The PBL version of the model predicts a larger value of $\Delta S$ and again gives a better agreement with the observational data than the surface-layer version of the model.

\section{Summary}

A Non-Linear Mixed Spectral Finite-Difference model for neutral planetary boundary-layer flow over complex terrain has been developed. The model uses $E-\ell$ turbulence closure. Some of early limitations on the surface-layer version of model are removed and model can simulate flows that are more representative of the real atmosphere. This new model has good potential in wind energy applications.

Acknowledgements. This research has been funded by grants from Mathematics for Information Technology and Complex Systems (MITACS) and Zephyr North of Canada.

Edited by: E. Batchvarova

Reviewed by: A. Beljaars and another anonymous referee

\section{SC $\mid$ nat $^{\mathbf{T}} \begin{aligned} & \text { The publication of this article is sponsored } \\ & \text { by the Swiss Academy of Sciences. }\end{aligned}$}

\section{References}

Ayotte, K. W. and Taylor, P. A.: A mixed spectral finite-difference 3D model of neutral planetary boundary-layer flow over topography, J. Atmos. Sci., 50, 3523-3537, 1995.

Ayotte, K. W., Xu, D. , and Taylor, P. A.: The impact of turbulence clousre schemes on predictions of the mixed spectral finitedifference model of flow over topography, Bound.-Lay. Meteorol., 68, 1-33, 1994.

Beljaars, A. C. M., Walmsley, J. L., and Taylor, P. A.: A mixed spectral finite-difference model of neutrally stratified boundarylayer flow over roughness changes and topography, Bound.-Lay. Meteorol., 38, 273-303, 1987.

Karpik, S. R.: An improved method for integrating the mixed spetral finite difference (MSFD) model equations, Bound.-Lay. Meteorol., 43, 273-286, 1988.

Walmsley, J. L. and Taylor, P. A.: Boudnary-layer flow over topography: impacts of the Askervein study, Bound.-Lay. Meteorol., 78, 291-320, 1996.

Weng, W. and Taylor, P. A.: On modelling the one-dimensional atmospheric boundary layer, Bound.-Lay. Meteorol., 107, 371400, 2003.

Weng, W., Chan, L., Taylor, P. A., and Xu, D.: Modelling stably stratified boudnary-layer flow over low hills, Q. J. Roy. Meteorol. Soc., 123, 1841-1866, 1997.

$\mathrm{Xu}, \mathrm{D}$. and Taylor, P. A.: A non-linear extension of the mixed spectral finite-difference model for neutrally stratified turbulent flow over topography, Bound.-Lay. Meteorol., 59, 177-186, 1992.

$\mathrm{Xu}$, D., Ayotte, K. W., and Taylor, P. A.: Development of the NLMSFD model of turbulent boundary-layer flow over topography, Bound.-Lay. Meteorol., 70, 341-367, 1994. 